\title{
Application of element decomposing method for solving integer problems
}

\author{
Ekkaphon Jaiyen ${ }^{1,}{ }^{*}$ and Komgrit Leksakul ${ }^{2}$ \\ ${ }^{1}$ Ph.D.'s Degree Program in Industrial Engineering, Department of Industrial Engineering, Faculty of Engineering, Chiang Mai \\ University, Chiang Mai 50200, Thailand \\ ${ }^{2}$ Department of Industrial Engineering, Faculty of Engineering, Chiang Mai University, Chiang Mai 50200, Thailand
}

\begin{abstract}
This research objective is to solve the integer problems by using an algorithm that applied to the element decomposing method (EDCM). Integer problem is a NP-hard when the problem is large-size, more time needed to solve the problem. The EDCM cuts a structure into several elements and reconnects elements at "nodes". This process can disconnect other nodes that not connected with the element. Moreover, it appropriately need in optimization solutions for applications, in which can give answers faster by cutting the nodes in finding the answers. There's 2 phases of step on this research. The first phase is input data and simplex method, while the second phase is creating and developing the algorithm from EDCM application. The comparison results show how two methods are carried out between EDCM and B\&B Method. The results from two methods are focused on the value and solution step by step. According to the problem, it can be solved within the number of variables 2-15. The value of the difference in the answer is on average at $0.00 \%$ and solution step used for solving problem is less on average at $40.89 \%$.
\end{abstract}

\section{Introduction}

Operation Research (OR) is using of the model in mathematics, statistics and algorithm to help in decision making. This will bring to use much in the business, the industry, the government sector etc. The function to search for the practice that will give the best result (Search for Optimality) will be the main idea. The operation research has come into existence during the period of the World War II, the military administration of British has given the scientist team to study research about strategy and tactic in protecting the country for both on the road and the air [1].

Integer linear programming (ILP) is main part of mathematical programing model that is NP-Hard problem. Integer liner programming is optimal solution in linear programming to the integer number, in which the objective function and constraint functions are linear. ILP consist production planning problem, capital budgeting, fixed-change problem, scheduling problem, telecommunications network problem etc.

Algorithm is the sequence of the procedure in solving the problem to solution to find the best answer or this is closed to the complexity and the difficulty. Algorithm can be used to solve the integer linear programming that is consist of 2 types, the exact method and the heuristic method. For both of 2 types, this will be different in the viewpoint of the quality of the answer and the solution time. If this has used the exact method in solving the problem, it would have made to get the best answer but it would have used the long time period. Heuristic techniques are powerful and flexible search methodologies that have successfully tackled practical difficult problems. Heuristic and meta-heuristic algorithms are used to produce good-quality solutions in reasonable amount of computation times and acceptable for practical purposes.

In solving the integer problem, it consists of 2 methods, which is a popular method that are consist of the branch and bound (B\&B) method and the cutting plane method. B\&B method is widely popular method, which uses tools for solving large-scale (NP-hard) combinatorial optimization problems. The B\&B algorithm operates according to two principles: (1) It recursively splits the search space into smaller spaces, thus minimizing the function on those smaller spaces; the splitting is called branching. (2) Branching alone would amount to brute-force enumeration of candidate solutions and the testing of them all. In other part, cutting plane method is an alternative to the branch and bound method, which also can be used to solve integer problems. The fundamental idea behind cutting planes is to add constraints to linear program until the feasible optimal basic solution takes on integer values. On contrary, this method has to be carefully performed as it has constraints, and one would not want to change the problem because of the addition of the constraints. This method will add a special type of constraint called a cut. $[2,3]$ In both methods, the constraints added will eliminate the portions of relaxed solution space, but never any of the feasible integer points. Neither of the two methods can be claimed to be uniformly more

\footnotetext{
* Corresponding author: bang0285@gmail.com
} 
effective in solving ILP's. Nevertheless, B\&B methods are computationally far more successful than the cuttingplane methods. For this reason, most of commercial codes are based on the use of the $\mathrm{B} \& \mathrm{~B}$ procedure. However, the explicit enumeration is normally impossible due to the exponentially increasing number of potential solutions. The use of bounds for optimizing the function, combined with the value of the current best solution enables the algorithm to search parts of the solution space implicitly [4]. Therefore, when there are many variables in the solution, the time and number of branches required are more. This method takes longer time to process the solution.

Finite element method (FEM) is a numerical technique to finds approximate solutions to a boundary value problem for partial differential equations, which now comprehensively used in solid and structural mechanics $[5,6]$. The method of The FEM is a mathematical method that brought to be applied in software application creation, used for calculation to solve the engineering problem. The Finite element method is done by filling the shape of the product with the small piece that has geometric form. In FEM, the structural system is modelled by a set of appropriate finite elements interconnected at a point called "nodes". The elements might have physical properties, such as thickness, density, Young's modulus, shear modulus, Poisson's ratio and etc.

Currently, from the problem in using the algorithm that has applied to solve the mathematic problem. This has still the limitation and this will use the time period in solution the answer for a long time. This will have the idea in applying to use the method in the operation research in creating and developing the new algorithm for solving the problem to will be faster further.

\section{Research Methodology}

The research study aims to solve an optimization problem using an algorithm that applies the decomposed element method, as shown in Figure 1.

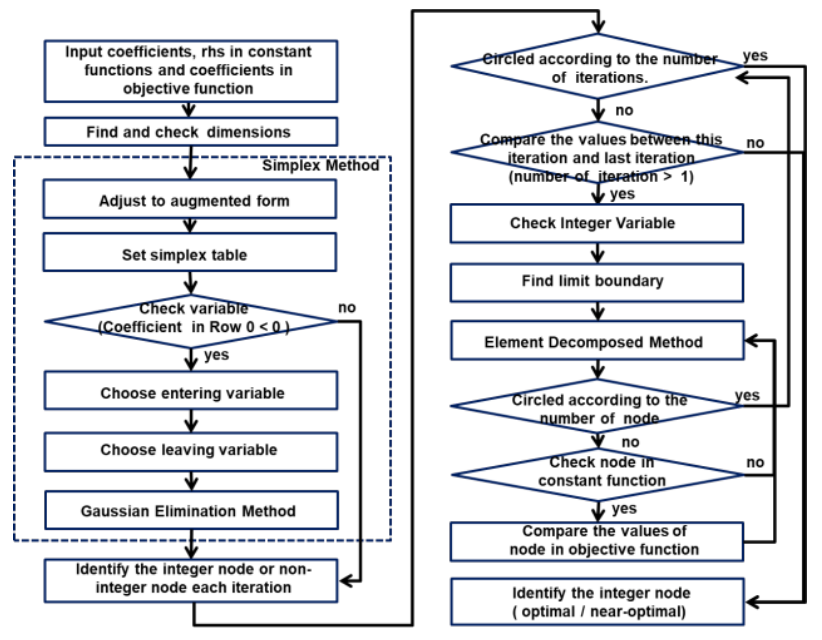

Figure 1. Steps of the research methodology.
This research study has two phases. The first phase consists of the input data and simplex method. The second phase is about creating and developing the algorithm via application of the element decomposing method (EDCM).

Phase 1; consists of the input data and simplex method, which is performed as follows.

Step 1.1; The input coefficients, the right-hand side (RHS) in constant functions and coefficients in the objective function follow the mathematical equation form.

Example; Integer problem in 2 variables solved by graph method that is shown figure 2 .

Objective function: $\max \mathrm{z}=4 \mathrm{x}_{1}+3 \mathrm{x}_{2}$

Constant function : $\quad 15 \mathrm{x}_{1}+9 \mathrm{x}_{2} \leq 85$

$3 \mathrm{x}_{1}+4 \mathrm{x}_{2} \leq 24$

$\mathrm{x}_{1} \geq 0, \mathrm{x}_{2} \geq 0$

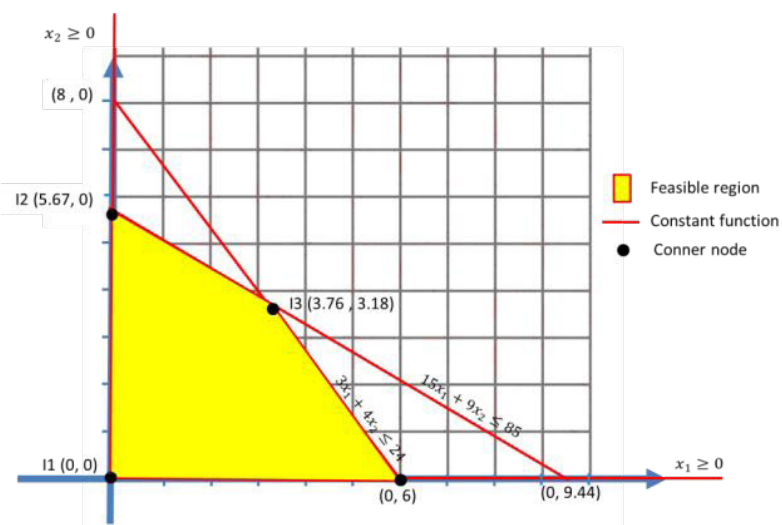

Figure 2. Feasible region of example from graph method

$$
\begin{array}{ll}
\text { Coefficient of objective function } & =\left[\begin{array}{ll}
4 & 3
\end{array}\right] \\
\text { Coefficient of constant functions } & =\left[\begin{array}{lll}
15 & 9 ; 3 & 4
\end{array}\right] \\
\text { Rhs. of constant functions } & =\left[\begin{array}{ll}
85 ; 24
\end{array}\right]
\end{array}
$$

Step 1.2; Finding examined according to the following conditions.

1. number of column in coefficients of objective function and coefficients of constant functions is equal.

2. number of row in coefficients of objective function and Rhs. of constant functions is equal.

Subsequently, write all the values of the coefficient of objective function, coefficient of the constant functions, and the right-hand side of the constant functions in a simplex table adjust to the augmented form for prepare to simplex method.

Step 1.3; Solving the problem using simplex method which is shown the node each iteration in Table 1.

Table 1. Node of Example Problem from Simplex Method.

\begin{tabular}{|c|c|c|c|}
\hline Iteration & $\mathbf{X}_{\mathbf{1}}$ & $\mathbf{X}_{\mathbf{2}}$ & $\mathbf{Z}$ \\
\hline Iteration 1 (i1) & 0 & 0 & 0 \\
\hline Iteration 2 (i2) & 5.67 & 0 & 22.67 \\
\hline Iteration 3 (i3) & 3.76 & 3.18 & 24.58 \\
\hline
\end{tabular}


Phase 2; consists of creating and developing the algorithm via application of the element decomposed method (EDCM) which is performed as follows.

Step 2.1; Using nodes from phase 1 to check the integer number in each iteration that starts from last iteration (Iteration 3; i3).

According to Example, the answer is that the optimal value $(z)=24.58$ is at node $\mathrm{i} 3$ which is $(\mathrm{x} 1, \mathrm{x} 2)=(3.76$, 3.18). However, if it is a pure integer problem, it is necessary to use the element decomposing method (EDCM) for solving the problem. In this method, the feasible region is divided into small sub-areas such as those of the integer unit, or it is the feasible region which has the Conner node as the integer node, as shown in Figure 3.

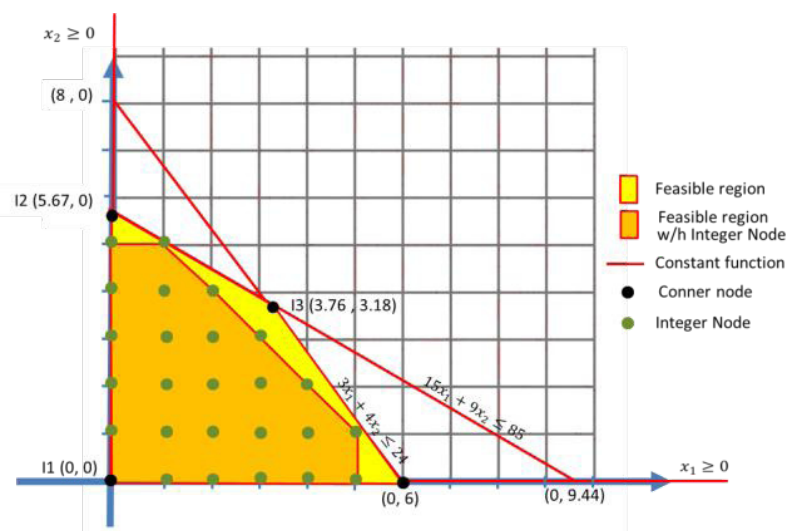

Figure 3. The feasible region with the integer problem

Step 2.2; Using the nodes in each iteration by making them adjust to integer node by using the element decomposing method (EDCM) which has three cases, namely Case 1 which is the complete integer node, Case 2 which is the non-complete integer node and Case 3 which is the non-integer node. In Case 2, the noncomplete integer node (Node form iteration 2) and Case 3 , the non-integer node (Node form iteration 3) adjust to the integer node by using the element decomposing method (EDCM), which is shown in Figure 4. The number of integer nodes is four, $2^{\wedge}$ number of variable.

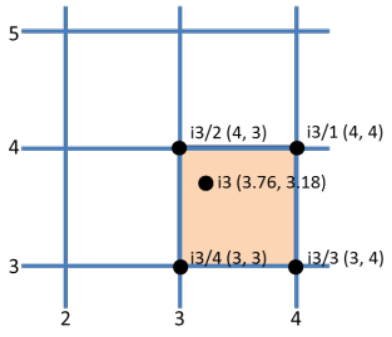

(A) Node form Iteration 3

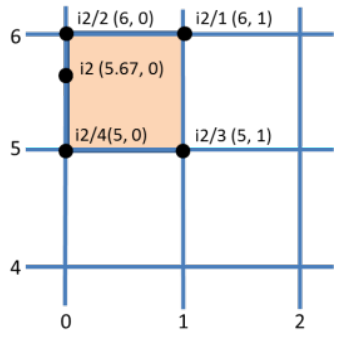

(B) Node from Iteration 2
Table 2. Integer Node by Using EDCM.

\begin{tabular}{|c|c|c|c|c|c|}
\hline Iteration & $\begin{array}{c}\text { Original } \\
\text { Node }\end{array}$ & Node 1 & Node 2 & Node 3 & Node 4 \\
\hline $\begin{array}{c}\text { Iteration 2 } \\
\text { (i2) }\end{array}$ & $\mathrm{i} 2$ & $\mathrm{i} 2 / 1$ & $\mathrm{i} 2 / 2$ & $\mathrm{i} 2 / 3$ & $\mathrm{i} 2 / 4$ \\
$(5.67,0)$ & $(6,1)$ & $(6,0)$ & $(5,1)$ & $(5,0)$ \\
\hline $\begin{array}{c}\text { Iteration 3 } \\
\text { (i3) }\end{array}$ & $\mathrm{i} 1$ & $\mathrm{i} 1 / 1$ & $\mathrm{i} 1 / 2$ & $\mathrm{i} 1 / 2$ & $\mathrm{i} 1 / 4$ \\
$(3.76,3.18)$ & $(4,4)$ & $(4,3)$ & $(3,4)$ & $(3,3)$ \\
\hline
\end{tabular}

Step 2.3; Checking the integer node in each of the constant functions. This starts from the node arranged in Step 2.2, as shown in Figure 5.

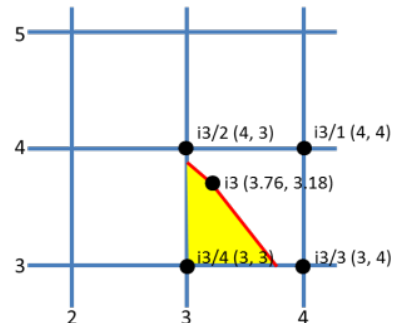

(A) Node form Iteration 3

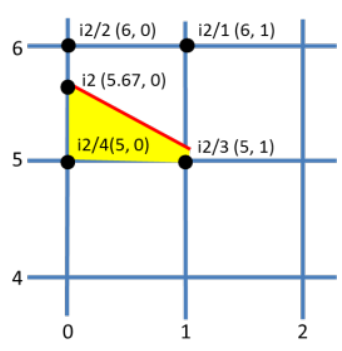

(B) Node from Iteration 2
Figure 5. The integer node after checking constant function.

In iteration 3 has node $i 3 / 1$, node $i 3 / 2$ and node $i 3 / 3$ which cannot pass the condition of the constraint function but node $i 3 / 4$ pass the conditions of both of the constraint functions. In iteration 2 has node $i 2 / 1$ and node $i 2 / 2$ which cannot pass the condition of the constraint function but node $i 2 / 3$ and node $i 2 / 4$ pass the conditions of both of the constraint functions.

Step 2.4; Finding the value in the objective function using the integer node after checking the constant function (from Step 2.3).

Checking all the points may need a lot of time, so there are steps for checking and finding the answers in order to reduce the length of time, as shown in Figure 6.

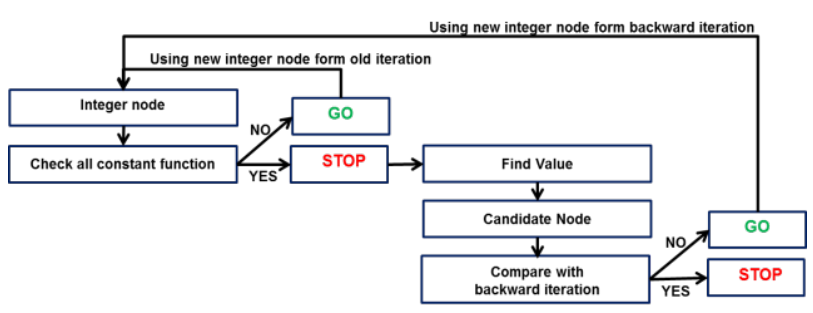

Figure 6. Step for checking node each iteration

The node from the last iteration is firstly considered for use in checking the constraint function (checking the feasible region) under the condition that it can pass all the constraint functions. After that, the value from the objective function is calculated. When the node passes the condition and stops at that step in the rest of the nodes in that iteration, the node from the checking acts as the candidate of that iteration.

The first node of each iteration, in the cases of both the non-integer node and the non-complete integer node, is out of the feasible region, or it cannot pass the constraint function of at least one equation, such as Node i3 and Node i2.

After that, a comparison is made between the value in one iteration and that in the next iteration. If the result shows the value to be greater than that of the Maximum Problem or less than that of the Minimum Problem, examining for the answer to this problem must be stopped. If the result does not show, it is necessary to get the candidate of the new node in the next iteration of checking, as shown in Figure 7. 


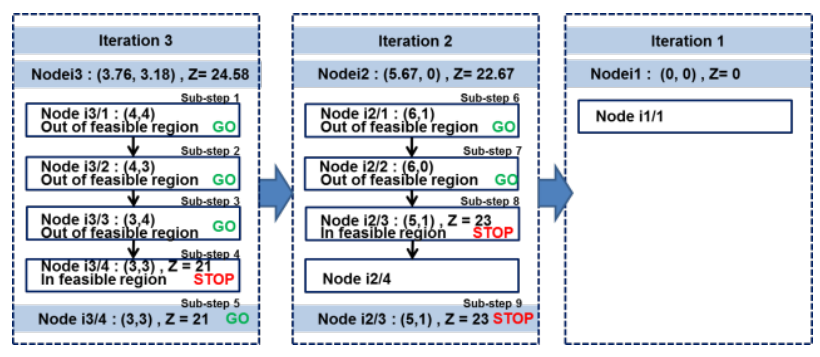

Figure 7. Step for checking the node and the finding value.

In example problem and Figure 7, it starts from Nodei3/1 (Iteration 3) as $(\mathrm{x} 1, \mathrm{x} 2)=(4,4)$.

Sub-step 1, it starts from Nodei3/1 as $(\mathrm{x} 1, \mathrm{x} 2)=(4,4)$ which cannot pass the condition of the constraint function no. $115^{*}(4)+9 *(4)=96>85$. (Go to consider node i3/2).

Sub-step 2, consider Nodei3/2 as $(\mathrm{x} 1, \mathrm{x} 2)=(4,3)$ which cannot pass the condition of the constraint function no. 1. (Go to consider node i3/3).

Sub-step 3, consider Nodei3/3 as $(\mathrm{x} 1, \mathrm{x} 2)=(3,4)$ which cannot pass the condition of the constraint function no. 2. (Go to consider node i3/4).

Sub-step 4, consider Node $3 / 4$ as $(x 1, x 2)=(3,3)$ which can pass the conditions of both of the constraint functions and possess the value $(\mathrm{z})=21$. After that, stop the examination at iteration 3 , which would make Node $3 / 4$ become the candidate of the iteration 3. (Stop consider in iteration 3 ).

Sub-step 5, consider the value from Node i3/4 in comparison to that from Node i2 and determine that Node i3/4 is less than Node i2 $(21<22.67)$ into consider the new node in the next iteration. (Go to consider iteration 2).

Sub-step 6, it starts from Nodei2/1 (Iteration 2) as $(\mathrm{x} 1, \mathrm{x} 2)=(6,1)$ which cannot pass the condition of the constraint function no. 1. (Go)

Sub-step 7, consider Nodei2/2 as $(\mathrm{x} 1, \mathrm{x} 2)=(6,0)$ which cannot pass the condition of the constraint function no. 1. (Go)

Sub-step 8, consider Node $2 / 3$ as $(\mathrm{x} 1, \mathrm{x} 2)=(5,1)$ which can pass the conditions of both of the constraint functions and possess the value $(\mathrm{z})=23$. After that, stop the examination at iteration 2, which would make Node $2 / 3$ become the candidate of the iteration 2. (Stop in iteration 2)

Sub-step 9, consider the value from Node i2/3 in comparison to that from Node i1 and determine that Node i2/3 is higher than Node il $(23>0)$. Then, stop the examination for the answer to the problem when the answer is at Node $i 2 / 3$ as $(x 1, x 2)=(5,1)$ and value $(z)=$ 23. (Stop this solution)

\section{Result and Discussion}

The comparison of results is carried out between the results from algorithm obtained by the application of the element decomposed method (EDCM) and the results from exact solution (Branch and bound method; B\&B).

The results from two methods are focusing on values and solution step, as shown in table 3. EDCM count number of node from decomposing and $\mathrm{B} \& \mathrm{~B}$ count number of branch. According to the problem, it can be solved with the number of variables that are 2, 3, 6 and 9. These results are comparing by percentages of gap in terms of value and solution step, as shown in table 3 .

Table 3. Values and solution step

\begin{tabular}{|l|c|c|c|c|c|}
\hline \multirow{2}{*}{$\begin{array}{c}\text { Problem } \\
\text { (NO. of variable) }\end{array}$} & \multicolumn{2}{|c|}{ Value } & \multicolumn{3}{c|}{ Solution Step } \\
\cline { 2 - 6 } & B\&B & EDCM & B\&B & EDCM & $\begin{array}{c}\text { Percentages } \\
\text { of gap }\end{array}$ \\
\hline Example (2) & 23 & 23 & 13 & 7 & $-46.15 \%$ \\
\hline Telfa Problem (2) & 40 & 40 & 7 & 3 & $-57.14 \%$ \\
\hline Dorian Auto (3) & 6000 & 6000 & 11 & 4 & $-63.64 \%$ \\
\hline Food Choices (6) & 57 & 57 & 15 & 14 & $-6.67 \%$ \\
\hline S.Confederation (9) & 633250 & 633250 & 15 & 6 & $-60.00 \%$ \\
\hline Stein (15) & 9 & 9 & 17 & 15 & $-11.76 \%$ \\
\hline
\end{tabular}

Based on Table 3, the results are solved by B\&B method and EDCM that can resolve problems for 2 to 15 variables and shows the percentages of difference in terms of solution step and solution time when a comparison is made between the B\&B method and EDCM. Value of the difference in the answer is at an average of $0.00 \%$ (no difference) and in the solution step used for solving the problem is at an average of $40.89 \%$. B\&B and EDCM can solve small problem less than 1 second. From the result of the small problem, this will show the difference of the solution step in solving the integer problem clearly. This will lead to the large size problem more.

The authors world like to gratefully acknowledge Department of Industrial Engineering, Chiang Mai University, for the supporting of this research work. Financial support from the Thailand Research Fund through the Research and Researchers for Industries Project (Grant NO. PHD59I0002) to Mr. Ekkaphon Jaiyen and Assoc. Prof. Dr. Komgrit Leksakul.

\section{References}

[1] Joseph F. M., (1987) OR Forum-British Operational Research in World War II. Operations Research 35(3):453-470.

[2] Michael, A. T., (2014), Cutting Plane Techniques, available at: http://mat.gsia.cmu.edu /orclass/integer /node14.html. (accessed 8 April 2016).

[3] Lewis, F. D., (1999), Cutting Plane Techniques,available at: http://www.cs.uky.edu/ lewis/ cs-heuristic/text/integer/cutting.html. (accessed 8 April 2016).

[4] Jens, C., (1999), Branch and Bound Algorithms Principles and Examples, available at: http://janders.eecg.toronto.edu/1387/readings/b_and_b.p df. (accessed 18 January 2016).

[5] Thomas, J. H., Wing K., and Alec B., (1979), Finite Element Analysis of Incompressible Viscous Flows by the Penalty Function Formulation, JOURNAL OF COMPUTATIONAL PHYSICS, Vol 30, pp.1-60.

[6] Charles, E. K., The Finite Element Method in Mechanical Design, Virginia Polytechnic Institute and State University, Boston: PWS-KENT Publishing Company, pp. 1-7. 